\title{
Modified Ratio Estimator in Simple Random Sampling using Auxiliary Information
}

\author{
Sumaira Ajmal Khan
}

\author{
Hafsa Abbas
}

Mehran Faiz

Lubna Shaheen

\begin{abstract}
In this study, we offer a new estimator for population variance in simple random sampling based on auxiliary information. We calculated the proposed estimator's bias and MSE equations and compared them to the bias and MSE of existing estimators, demonstrating that the new estimator is more efficient than the existing estimators proposed by different authors. With the aid of numerical example, we can support this theoretical result.
\end{abstract}

\section{Keywords}

Variance estimator, bias, MSE, simple random sampling, auxiliary information, efficiency

\section{INTRODUCTION}

Auxiliary information has been used extensively in estimation of parameters like mean and variance since several decades. Isaki (1983) [1] got inspiration from ratio estimator of finite population mean and proposed a ratio estimator, usually called classical estimator, of finite population variance. But Singh and Solanki (2013a) claimed that Isaki (1983) [1] ratio estimator is the member of the class of estimators developed by Das and Tripathi (1978) [3]. Arcos and Rueda (1997) [4] suggested multivariate ratio estimator for population variance. Ahmed et al. (2000) [5] criticized the claim of Arcos and Rueda (1997) [4], Kadilar and Cingi (2006a) [6] developed an estimator, ratio-type estimator of the mean of population, Kadilar and Cingi (2006b) [7] extended the idea of Isaki (1983) [1] ratio estimator for population variance. Kadilar and Cingi (2006b) [7] involved the information available about coefficient of variation and coefficient of kurtosis of the auxiliary variable to generate these estimators under simple random sampling as well. Gupta and Shabbir (2008) [8] gave a hybrid class of variance estimators of population mean. Subramani and Kumarapandiyan (2012a) [9] modified the usual ratio-type estimator of Kadilar and Cingi (2006b) [7] for population variance using population median obtained from auxiliary variable. Subramani and Kumarapandiyan (2012b) [10] further modified the usual ratio-type variance estimators using lower and upper quartiles, inter-quartile range, quartile deviation and quartile average of the auxiliary variable. Subramani and Kumarapandiyan (2013) [11] developed another more efficient modified ratio-type estimator using median and coefficient of variation of the auxiliary variable.

\section{NOTATION}

Before delving deeper into the classical ratio type variance estimator, modified ratio type variance estimators, and proposed modified ratio type variance estimator, the notations that will be used in this paper are listed below:

1. Population size

N

2. Sample size
3. Population mean of the study variable $\mathrm{y}$

$$
\bar{Y}=\frac{\sum_{i=1}^{N} y_{i}}{N}
$$

4. Population mean of the auxiliary variable $\mathrm{x}$

$$
\bar{X}=\frac{\sum_{i=1}^{N} x_{i}}{N}
$$

5. Sample mean of the study variable y

$$
\bar{y}=\frac{\sum_{i=1}^{n} y_{i}}{n}
$$

6. Sample mean of the auxiliary variable $\mathrm{x}$

$$
\bar{x}=\frac{\sum_{i=1}^{n} x_{i}}{n}
$$

7. Population variance of the study variable $y$

$$
S_{Y}^{2}=\frac{\sum_{i=1}^{N}\left(y_{i}-\bar{Y}\right)^{2}}{N-1}
$$

8. Population variance of the auxiliary variable $\mathrm{x}$

$$
S_{X}^{2}=\frac{\sum_{i=1}^{N}\left(x_{i}-\bar{X}\right)^{2}}{N-1}
$$

9. Sample variance of the study variable $\mathrm{y}$

$$
S_{y}^{2}=\frac{\sum_{i=1}^{n}\left(y_{i}-\bar{y}\right)^{2}}{n-1}
$$

10. Sample variance of the auxiliary variable $\mathrm{x}$

$$
S_{x}^{2}=\frac{\sum_{i=1}^{n}\left(x_{i}-\bar{x}\right)^{2}}{n-1}
$$

11. Coefficient of variation

$$
C_{x}=\frac{S_{x}}{\bar{X}}, \quad C_{y}=\frac{S_{y}}{\bar{Y}}
$$

12. Skewness of the auxiliary variable

$$
\beta_{1(x)}=\frac{\mu_{03}^{2}}{\mu_{02}^{2}}
$$

13. Kurtosis of the auxiliary variable

$$
\beta_{2(x)}=\frac{\mu_{04}^{2}}{\mu_{02}^{2}}
$$

14. Kurtosis of the study variable

$$
\beta_{2(y)}=\frac{\mu_{40}^{2}}{\mu_{20}^{2}}
$$

15. $\gamma=\frac{(1-f)}{n}$ 


\section{SOMEEXISTINGESTIMATORS}

\section{Isaki (1983)}

Motivated by the estimator of the population mean

$$
\hat{\mu}_{y R}=\frac{\bar{y}}{\bar{x}} \bar{X}
$$

Isaki (1983) [1] suggested the ratio estimators of the variance population using auxiliary information.

$$
t_{\text {Isaki }}=s_{y}^{2} \frac{S_{x}^{2}}{s_{x}^{2}}
$$

The MSE of the above estimator using first order approximation is

$$
\operatorname{MSE}\left(t_{\text {Isaki }}\right) \cong \lambda^{\prime} S_{y}^{4}\left\{\beta_{2}(y)+\beta_{2}(x)-2 h\right\}
$$

Or

$$
\operatorname{MSE}\left(t_{\text {Isaki }}\right) \cong \lambda^{\prime} S_{y}^{4}\left\{\beta_{2}^{\prime}(y)+\beta_{2}^{\prime}(x)-2 h^{\prime}\right\}
$$

And the bias is

$$
\operatorname{Bias}\left(t_{\text {Isaki }}\right) \cong \lambda^{\prime} S_{y}^{2}\left\{\beta_{2}(x)-h\right\}
$$

Or

$$
\operatorname{Bias}\left(t_{I s a k i}\right) \cong \lambda^{\prime} S_{y}^{2}\left\{\beta_{2}^{\prime}(x)-h^{\prime}\right\}
$$

Or

$$
\operatorname{Bias}\left(t_{\text {Isaki }}\right) \cong-\operatorname{Cov}\left(\frac{s_{y}^{2}}{s_{x}^{2}}, S_{x}^{2}\right)
$$

\section{Kadilar and Cingi (2005)}

Kadilar and Cingi(2005) [2] proposed the following class of estimators for population variance:

$$
\begin{gathered}
t_{C H 1}=s_{y}^{2}\left[s_{x}^{2}-\beta_{2}(x)\right]^{-1}\left[S_{x}^{2}-\beta_{2}(x)\right] \\
t_{C H 2}=s_{y}^{2}\left[s_{x}^{2} C_{x}-\beta_{2}(x)\right]^{-1}\left[S_{x}^{2} C_{x}-\beta_{2}(x)\right] \\
t_{C H 3}=s_{y}^{2}\left(s_{x}^{2}-C_{x}\right)^{-1}\left(S_{x}^{2}-C_{x}\right) \\
t_{C H 4}=s_{y}^{2}\left[s_{x}^{2} \beta_{2}(x)-C_{x}\right]^{-1}\left[S_{x}^{2} \beta_{2}(x)-C_{x}\right]
\end{gathered}
$$

The MSE's of the above estimators are given below respectively:

$$
\begin{array}{r}
M S E\left(t_{C H 1}\right) \cong \lambda^{\prime} S_{y}^{4}\left[\beta_{2}^{\prime}(y)-2 \frac{S_{x}^{2}}{S_{x}^{2}-\beta_{2}(x)} h^{\prime}\right. \\
\left.+\left\{\frac{S_{x}^{2}}{S_{x}^{2}-\beta_{2}(x)}\right\}^{2} \beta_{2}^{\prime}(x)\right] \\
M S E\left(t_{C H 2}\right) \cong \lambda^{\prime} S_{y}^{4}\left[\beta_{2}^{\prime}(y)-2 \frac{S_{x}^{2}}{S_{x}^{2} C_{x}-\beta_{2}(x)} h^{\prime}+\right. \\
\left.\left\{\frac{S_{x}^{2}}{S_{x}^{2} C_{x}-\beta_{2}(x)}\right\}^{2} \beta_{2}^{\prime}(x)\right]
\end{array}
$$

$$
\begin{aligned}
\operatorname{MSE}\left(t_{C H 3}\right) \cong \lambda^{\prime} S_{y}^{4} & {\left[\beta_{2}^{\prime}(y)-2 \frac{S_{x}^{2}}{S_{x}^{2}-C_{x}} h^{\prime}\right.} \\
& \left.+\left\{\frac{S_{x}^{2}}{S_{x}^{2}-C_{x}}\right\}^{2} \beta_{2}^{\prime}(x)\right]
\end{aligned}
$$

$$
\begin{aligned}
M S E\left(t_{C H 4}\right) \cong \lambda^{\prime} S_{y}^{4} & {\left[\beta_{2}^{\prime}(y)-2 \frac{S_{x}^{2}}{S_{x}^{2} \beta_{2}(x)-C_{x}} h^{\prime}\right.} \\
& \left.+\left\{\frac{S_{x}^{2}}{S_{x}^{2} \beta_{2}(x)-C_{x}}\right\}^{2} \beta_{2}^{\prime}(x)\right]
\end{aligned}
$$

The Biases of above estimators are given below respectively:

$$
\begin{gathered}
\operatorname{Bias}\left(t_{C H 1}\right) \cong \lambda^{\prime} S_{y}^{2} \frac{S_{x}^{2}}{S_{x}^{2}-\beta_{2}(x)}\left[\frac{S_{x}^{2}}{S_{x}^{2}-\beta_{2}(x)} \beta_{2}^{\prime}(x)-h^{\prime}\right] \\
\operatorname{Bias}\left(t_{C H 2}\right) \cong \lambda^{\prime} S_{y}^{2} \frac{S_{x}^{2}}{S_{x}^{2} C_{x}-\beta_{2}(x)}\left[\frac{S_{x}^{2}}{S_{x}^{2} C_{x}-\beta_{2}(x)} \beta_{2}^{\prime}(x)-h^{\prime}\right] \\
\operatorname{Bias}\left(t_{C H 3}\right) \cong \lambda^{\prime} S_{y}^{2} \frac{S_{x}^{2}}{S_{x}^{2}-C_{x}}\left[\frac{S_{x}^{2}}{S_{x}^{2}-C_{x}} \beta_{2}^{\prime}(x)-h^{\prime}\right] \\
\operatorname{Bias}\left(t_{C H 4}\right) \cong \lambda^{\prime} S_{y}^{2} \frac{S_{x}^{2}}{S_{x}^{2} \beta_{2}(x)-C_{x}}\left[\frac{S_{x}^{2}}{S_{x}^{2} \beta_{2}(x)-C_{x}} \beta_{2}^{\prime}(x)-h^{\prime}\right]
\end{gathered}
$$

\section{PROPOSEDESTIMATOR}

Using the known value of the auxiliary variable's population mean, we introduced a new modified ratio type variance estimator.For population variance, the modified ratio type variance estimator is defined as follows:

$$
t_{\text {new }}=s_{y}^{2} \frac{\rho^{2} S_{x}^{2}+\bar{X}^{2}}{\rho^{2} s_{x}^{2}+\bar{X}^{2}}
$$

\section{Bias of the proposed estimator}

$$
\begin{gathered}
t_{\text {new }}=S_{y}^{2}\left(1+e_{0}\right) \frac{\rho^{2} S_{x}^{2}+\bar{X}^{2}}{\rho^{2} S_{x}^{2}\left(1+e_{1}\right)+\bar{X}^{2}} \\
t_{\text {new }}=S_{y}^{2}\left(1+e_{0}\right)\left\{1+\Omega e_{1}\right\}^{-1}
\end{gathered}
$$

Where

$$
\Omega=\frac{\rho^{2} S_{x}^{2}}{\rho^{2} S_{x}^{2}+\bar{X}^{2}}
$$

Expanding the expression by using Taylor's series

$$
\begin{gathered}
t_{\text {new }}=S_{y}^{2}\left(1+e_{0}\right)\left\{1-\Omega e_{1}+\left(\Omega e_{1}\right)^{2}-\left(\Omega e_{1}\right)^{3}+\cdots\right\} \\
t_{\text {new }}=S_{y}^{2}\left(1+e_{0}\right)\left\{1-\Omega e_{1}+\left(\Omega e_{1}\right)^{2}\right\} \\
t_{\text {new }}-S_{y}^{2}=S_{y}^{2}\left\{e_{0}-\Omega e_{1}+\left(\Omega e_{1}\right)^{2}-\Omega e_{0} e_{1}\right\} \\
E\left(t_{\text {new }}-S_{y}^{2}\right)=E\left[S_{y}^{2}\left\{e_{0}-\Omega e_{1}+\left(\Omega e_{1}\right)^{2}-\Omega e_{0} e_{1}\right\}\right] \\
B\left(t_{\text {new }}\right)=\Omega \lambda^{\prime} S_{y}^{2}\left\{\Omega \beta_{2(x)}^{\prime}-h^{\prime}\right\}
\end{gathered}
$$




\section{MSE of the proposed estimator}

Now comes to MSE of the estimator

$$
\operatorname{MSE}\left(t_{\text {new }}\right)=\left\{S_{y}^{2}\left(e_{0}-\Omega e_{1}\right)\right\}^{2}
$$

( $\square$ ignore higher order)

$$
\begin{gathered}
\operatorname{MSE}\left(t_{\text {new }}\right) \quad=S_{y}^{4} E\left\{\left(e_{0}^{2}\right)+\Omega^{2}\left(e_{1}^{2}\right)-2 \Omega\left(e_{0} e_{1}\right)\right\} \\
=S_{y}^{4}\left\{\lambda^{\prime} \beta_{2(y)}^{\prime}+\Omega^{2} \lambda^{\prime} \beta_{2(x)}^{\prime}-2 \Omega \lambda^{\prime} h^{\prime}\right\} \\
=\lambda^{\prime} S_{y}^{4}\left\{\beta_{2(y)}^{\prime}+\beta_{2(x)}^{\prime}\left(\Omega^{2}-2 \Omega \frac{h^{\prime}}{\beta_{2(x)}^{\prime}}\right)\right\} \\
=\lambda^{\prime} S_{y}^{4}\left\{\beta_{2(y)}^{\prime}+\beta_{2(x)}^{\prime}\left(\Omega^{2}-2 \Omega v^{\prime}\right)\right\} \quad \rightarrow(i)
\end{gathered}
$$

Where

$$
v^{\prime}=\frac{h^{\prime}}{\beta_{2(x)}^{\prime}}
$$

In order to minimize MSE, Differentiate (i) partially w.r.t $\Omega$ and equating to zero.

$$
\begin{gathered}
\frac{\partial}{\partial \Omega} \operatorname{MSE}\left(t_{\text {new }}\right)=0 \\
\lambda^{\prime} S_{y}^{4} \frac{\partial}{\partial \Omega}\left\{\beta_{2(y)}^{\prime}+\beta_{2(x)}^{\prime}\left(\Omega^{2}-2 \Omega v^{\prime}\right)\right\}=0 \\
\Omega=v^{\prime}
\end{gathered}
$$

So,

Where

$$
\begin{gathered}
=\lambda^{\prime} S_{y}^{4}\left\{\beta_{2(y)}^{\prime}-\beta_{2(x)}^{\prime} v^{\prime 2}\right\} \\
=\lambda^{\prime} S_{y}^{4} \beta_{2(y)}^{\prime}\left\{1-v^{\prime 2} \frac{\beta_{2(x)}^{\prime}}{\beta_{2(y)}^{\prime}}\right\} \\
=\lambda^{\prime} S_{y}^{4} \beta_{2(y)}^{\prime}\left\{1-\left(\frac{h^{\prime}}{\sqrt{\beta_{2(x)}^{\prime} \beta_{2(y)}^{\prime}}}\right)^{2}\right\} M S E\left(t_{\text {new }}\right) \\
=\lambda^{\prime} S_{y}^{4} \beta_{2(y)}^{\prime}\left\{1-\left(\rho^{\prime}\right)^{2}\right\}
\end{gathered}
$$

\section{NUMERICAL STUDY}

The execution of the proposed modified ratio type variance

\begin{tabular}{|c|c|c|c|c|}
\hline \multirow{2}{*}{$\begin{array}{l}\text { Estimator } \\
\mathrm{s}\end{array}$} & \multicolumn{4}{|c|}{ Bias } \\
\hline & Data 1 & Data 2 & Data 3 & Data 4 \\
\hline Isaki[1] & $\begin{array}{l}1282.9969 \\
6\end{array}$ & $\begin{array}{l}135.99347 \\
1\end{array}$ & $\begin{array}{l}10.87589 \\
32\end{array}$ & 367.35093 \\
\hline $\begin{array}{l}\text { Kadilar } \\
\text { and } \\
\text { Cingi[2] }\end{array}$ & $\begin{array}{l}1413.1211 \\
6\end{array}$ & $\begin{array}{l}136.16903 \\
7\end{array}$ & $\begin{array}{l}12.69804 \\
37\end{array}$ & $\begin{array}{l}367.80396 \\
5\end{array}$ \\
\hline $\begin{array}{l}\text { Kadilar } \\
\text { and } \\
\text { Cingi[2] }\end{array}$ & -138.3148 & $\begin{array}{l}- \\
157.98237\end{array}$ & $\begin{array}{l}33.32632 \\
51\end{array}$ & $\begin{array}{l}731.21715 \\
1\end{array}$ \\
\hline $\begin{array}{l}\text { Kadilar } \\
\text { and } \\
\text { Cingi[2] }\end{array}$ & $\begin{array}{l}1288.2034 \\
5\end{array}$ & $\begin{array}{l}136.00423 \\
9\end{array}$ & $\begin{array}{l}11.32844 \\
5\end{array}$ & $\begin{array}{l}367.40221 \\
8\end{array}$ \\
\hline $\begin{array}{l}\text { Kadilar } \\
\text { and } \\
\text { Cingi[2] }\end{array}$ & $\begin{array}{l}- \\
67.864364\end{array}$ & $\begin{array}{l}- \\
178.34129\end{array}$ & $\begin{array}{l}- \\
3.347314 \\
2 \\
\end{array}$ & -57.03954 \\
\hline $\begin{array}{l}\text { Proposed } \\
\text { Estimato }\end{array}$ & $\begin{array}{l} \\
241.92096\end{array}$ & $\begin{array}{l}- \\
784.70582 \\
\end{array}$ & $\begin{array}{l}- \\
3.362946 \\
\end{array}$ & $\begin{array}{l}- \\
77.110599 \\
\end{array}$ \\
\hline
\end{tabular}
estimator is surveyed with that of traditional ratio type estimator and existing modified ratio type variance estimators recorded in table 1 for certain common populations. The

\begin{tabular}{|c|c|c|c|c|}
\hline \multirow{2}{*}{$\begin{array}{c}\text { Estimator } \\
\mathrm{S}\end{array}$} & \multicolumn{4}{|c|}{ Mean Squared Error(MSE) } \\
\hline & Data 1 & Data 2 & Data 3 & Data 4 \\
\hline Isaki[1] & $\begin{array}{l}3778793.0 \\
28\end{array}$ & $\begin{array}{l}3579661 \\
1.55 \\
\end{array}$ & \begin{tabular}{|l}
3924.948 \\
201 \\
\end{tabular} & $\begin{array}{l}1438805.63 \\
7\end{array}$ \\
\hline $\begin{array}{l}\text { Kadilar } \\
\text { and } \\
\text { Cingi[2] }\end{array}$ & $\begin{array}{l}3983111.9 \\
77\end{array}$ & $\begin{array}{l}3579672 \\
0.6\end{array}$ & $\begin{array}{l}4249.507 \\
513\end{array}$ & $\begin{array}{l}1439774.89 \\
8\end{array}$ \\
\hline $\begin{array}{l}\text { Kadilar } \\
\text { and } \\
\text { Cingi[2] }\end{array}$ & $\begin{array}{l}2514073.3 \\
51\end{array}$ & $\begin{array}{l}3582387 \\
6.09\end{array}$ & $\begin{array}{l}8667.305 \\
927\end{array}$ & $\begin{array}{l}2300502.29 \\
5\end{array}$ \\
\hline $\begin{array}{l}\text { Kadilar } \\
\text { and } \\
\text { Cingi[2] }\end{array}$ & $\begin{array}{l}3786820.8 \\
36\end{array}$ & $\begin{array}{l}3579661 \\
8.23\end{array}$ & $\begin{array}{l}4003.905 \\
33\end{array}$ & $\begin{array}{l}1438915.34 \\
8\end{array}$ \\
\hline $\begin{array}{l}\text { Kadilar } \\
\text { and } \\
\text { Cingi[2] }\end{array}$ & $\begin{array}{l}7990304.8 \\
12\end{array}$ & $\begin{array}{l}5983987 \\
1.24\end{array}$ & $\begin{array}{l}3636.702 \\
702\end{array}$ & $\begin{array}{l}1557091.88 \\
4\end{array}$ \\
\hline $\begin{array}{l}\text { Proposed } \\
\text { Estimator }\end{array}$ & $\begin{array}{l}2614574.7 \\
28\end{array}$ & $\begin{array}{l}4533482 \\
5.03\end{array}$ & $\begin{array}{l}3750.092 \\
953\end{array}$ & $\begin{array}{l}1258334.94 \\
1\end{array}$ \\
\hline
\end{tabular}
population parameters and the constants computed are given below:
Table 1: Parameters and Constants of Populations

\begin{tabular}{|l|l|l|l|l|}
\hline & Data 1 & Data 2 & Data 3 & Data 4 \\
\hline $\boldsymbol{N}$ & 278 & 103 & 80 & 70 \\
\hline $\boldsymbol{n}$ & 30 & 40 & 20 & 25 \\
\hline$\overline{\boldsymbol{Y}}$ & 39.068 & 62.6212 & 51.8264 & 96.7000 \\
\hline$\overline{\boldsymbol{X}}$ & 25.111 & 556.5541 & 11.2646 & $\begin{array}{l}175.267 \\
1\end{array}$ \\
\hline $\boldsymbol{S}_{\boldsymbol{y}}$ & $\begin{array}{l}56.4571 \\
67\end{array}$ & 91.3549 & 18.3569 & 60.714 \\
\hline $\boldsymbol{S}_{\boldsymbol{x}}$ & $\begin{array}{l}40.6747 \\
\end{array}$ & 670.1643 & 8.4563 & 140.857 \\
\hline $\boldsymbol{\beta}_{\mathbf{2}}(\boldsymbol{y})$ & 25.8969 & 37.1279 & 2.2667 & 4.7596 \\
\hline $\boldsymbol{\beta}_{\mathbf{2}}(\boldsymbol{x})$ & 38.8898 & 17.8738 & 2.8664 & 7.0952 \\
\hline $\boldsymbol{h}$ & 26.8142 & 17.2220 & 2.2209 & 4.6038 \\
\hline $\boldsymbol{\rho}$ & 0.7213 & 0.7298 & 0.9413 & 0.7293 \\
\hline
\end{tabular}

The biases and mean squared errors of the existing and proposed modified ratio type variance estimator for the populations given above are given in the following tables:

Table 2: Biases of the existing and proposed modified ratio type variance estimator

Table 3: MSE of the existing and proposed modified ratio type variance estimators 
From Table 2, it is observed that the bias of the proposed modified ratio type

Variance estimator is less than the biases of the traditional and existing modified ratio type variance estimators. Similarly from Table 3, it is observed that the mean squared error of the proposed modified ratio type variance estimator is less than the mean squared errors of the traditional and existing modified ratio type variance estimators.

\section{CONCULSION}

In this article we have proposed a modified ratio type variance estimator using known value of population mean of auxiliary variable. The bias and mean squared error of the proposed modified ratio type variance estimator are obtained and compared with that of traditional ratio type variance estimator and existing modified ratio type variance estimators. We have also assessed the performances of the proposed estimator for some known populations. It is observed that the bias and mean squared error of the proposed estimator is less than the biases and mean squared errors of the traditional and existing estimators for certain known populations.

\section{REFERENCES}

[1] Isaki, C. T. (1983). Variance estimation using auxiliary information. Journal of the American Statistical Association, 78(381), 117-123.

[2] Kadilar, C., \& Cingi, H. (2005). A new ratio estimator in stratified random sampling. Communications in Statistics-Theory and Methods, 34(3), 597-602.

[3] Das, A. K. (1978). Use of auxiliary information in estimating the finite population variance. Sankhya, $c, 40$, 139-148.

[4] Cebrián, A. A., \& García, M. R. (1997). Variance estimation using auxiliary information: An almost unbiased multivariate ratio estimator. Metrika,45(1), 171-178.

[5] Ahmed, M. S., Raman, M. S., \& Hossain, M. I. (2000). Some competitive estimators of finite population variance using multivariate auxiliary information. International journal of information and management sciences, 11(1), 49-54.

[6] Kadilar, C., \& Cingi, H. (2006a). Improvement in variance estimation using auxiliary information. Hacettepe Journal of mathematics and Statistics, 35(1), 111-115.

[7] Kadilar, C., \& Cingi, H. (2006b). Ratio estimators for the population variance in simple and stratified random sampling. Applied Mathematics and Computation, 173(2), 1047-1059.

[8] Gupta, S., \& Shabbir, J. (2008). Variance estimation in simple random sampling using auxiliary information. Hacettepe Journal of mathematics and Statistics, 37(1), 57-67.

[9] Subramani, J., \& Kumarapandiyan, G. (2012a). Estimation of population mean using co-efficient of variation and median of an auxiliary variable. International Journal of Probability and Statistics, 1(4), 111-118.

[10] Subramani, J., \& Kumarapandiyan, G. (2012b). Variance Estimation Using Median of the Auxiliary Variable. International Journal of Probability and Statistics, 1(3), 36-40.

[11] Subramani, J., \& Kumarapandiyan, G. (2013). Estimation of variance using known coefficient of variation and median of an auxiliary variable. Journal of Modern Applied Statistical Methods, 12(1), 11. 